\title{
Numerical investigation on electroosmotic flow in a porous channel
}

\author{
T. A. Cheema, K. W. Kim, M. K. Kwak, C. Y. Lee, G. M. Kim, C.W. Park* \\ School of Mechanical Engineering, Kyungpook National University, 1370 Sankyuk-dong, Buk-gu, \\ Deagu, 702-701, S. Korea. \\ *Corresponding Author: chwoopark@knu.ac.kr
}

\begin{abstract}
Porous Electroosmotic (EO) pump is the type of pump which contains a porous media regime to perform flow moving under electric field application. It can use a constant value of porosity with constant pore diameter limiting the flow rates especially near the wall region in addition to the boundary layer effects. In this study, a composite porous layer has been proposed to use in EO pump with a high porosity value near the wall region rather than the central region of the flow field. A 2D rectangular porous channel of $4 \mathrm{~mm} \times 1.5 \mathrm{~mm}$ is employed and it has been divided vertically into three major domains of variable. As a result, an appreciable increase in velocity magnitude especially near wall region but also in the central region
\end{abstract}

Keywords: Electroosmotic, porosity, positional variation, wall region, central region

\section{Introduction}

The technological advancement and importance of porous media in a variety of industrial applications has made it a prominent research topic since last three decades. In the past, the focus was mainly to the development of theory related to porous media but recently, this trend has been shifted to performance enhancement of the applications involving the porous media. Porosity, permeability, morphological and geometrical configurations, thermal dispersion and local thermal non-equilibrium (LTNE) are some of the variables involved in the analysis to improve the performance of porous media previously ${ }^{(1,2)}$.

Electroosmotic driving flow was first observed by Reuss in 1809 during an experiment on porous clays. Since then it has been widely studied in the areas of electrochemistry, physics and vascular plant biology. There have been many numerical and experimental investigations of electroosmotic flow in porous media ${ }^{(3-6)}$. Electroosmotic pump is one such device which uses a porous membrane to exchange selective ions with the help of electroosmotic flow i.e. the motion of the liquid induced by an applied electric field ${ }^{(7)}$. Such kind of pump is used for drug delivery, cooling of microelectronic systems or for the fuel delivery in fuel cells.

Mechanical pressure is applied to assemble the devices having porous media as the central part which reduces pore diameter and hence the porosity is reduced near wall region leading to a reduced mass and flow properties. In order to counter and compensate the effects of reduced porosity near the wall region, a new method of positional variation of porosity is proposed to enhance the flow and mass transfer properties through porous media in an electroosmotic pump. The reduced flow and mass transfer abilities of the porous layers can be improved by introducing new composite porous layers having high porosity value in the outer region rather than in the central region of the porous media. In the present study, we have carried out a numerical investigation to observe electroosmotic pump flow features with variation of porosity.

\section{Model Development and Mathematical Modeling}

A two dimensional rectangular porous channel connected with two electrodes placed at bottom corners has been modeled as shown in Fig. 1. The cross section of channel is $4 \mathrm{~mm} \times 1.5 \mathrm{~mm}$ and it has been divided vertically into three major domains of variable and constant porosity region in order to implement the concept of a composite porous layer. The division of the porous media was done on the basis of boundary layer developed in the same porous layer with constant unit porosity. The outer region 
will take a higher porosity than the central region porosity value of 0.6 .

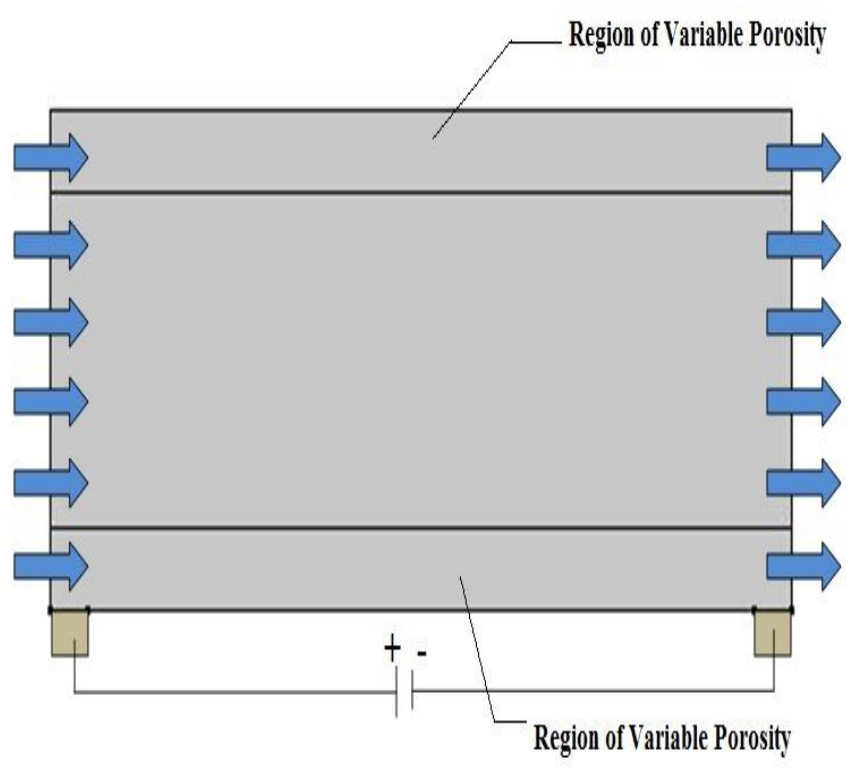

Fig. 1. Electroosmotic flow and porous domain model used in the study.

The computational domain was assumed to contain a single phase fluid, with density $\left(\rho=1000 \mathrm{~kg} / \mathrm{m}^{3}\right)$ and viscosity ( $\mu=1.0 \mathrm{E}-03 \mathrm{~Pa}-\mathrm{s})$. The effects of electrochemical reaction, compressibility and gravitation have been neglected and a laminar fluid flow was considered. The flow field has been investigated by using continuity equation for flow and current density as given below.

$$
\begin{gathered}
\nabla \cdot \vec{u}=0 \\
\nabla \cdot \vec{i}=0
\end{gathered}
$$

where, $\vec{u}$ is the velocity in $(\mathrm{m} / \mathrm{s})$ and $\vec{i}$ is the current density $\left(\mathrm{A} / \mathrm{m}^{2}\right)$.

The velocity can be evaluated using potential difference according to the relation:

$$
\vec{u}=\frac{\varepsilon_{p} \varepsilon_{w} \zeta}{\mu \tau} \nabla V
$$

where, $\varepsilon_{p}$ is the base porosity, $\varepsilon_{w}$, and $\xi$ are the fluid permittivity $(\mathrm{F} / \mathrm{m})$ and zeta potential $(\mathrm{V})$ respectively. $\tau$ denotes the tortuosity of the porous media and $\mathrm{p}$ and $\mathrm{V}$ are the applied pressure $(\mathrm{Pa})$ and voltage $(\mathrm{V})$.

Similarly the current density can be evaluated by using the following relation.

$$
\vec{i}=-\kappa \nabla V
$$

where, $\kappa$ denotes the conductivity $(\mathrm{S} / \mathrm{m})$.

All the boundaries have been assigned with an insulated condition except the electrodes having a $0.1 \mathrm{~V}$ and $0 \mathrm{~V}$ at anode and cathode surface respectively.

In this study, we used the commercial software COMSOL-Multiphysics, which uses a finite element method (FEM) to solve discritised equations. The two new slots introduced outside the central region were assigned to have a higher porosity ranging from $0.7 \sim 0.9$ than the central region having base porosity value of 0.6. The physics of electrosomotic flow in the porous channel have been implemented for a steady state simulation by using a PDE equation solver application mode available in the commercial code. The values of some of the important parameters used in this study have been listed in Table. 1.

The computational domain was discretized using triangular mesh with maximum element size of $0.1 \mathrm{~mm}$ to form 1538 elements and 6378 degrees of freedom were solved. It took 10 seconds to solve the computational domain on $2.93 \mathrm{GHz}$ computer processor. In each case Direct solver was used for 1000 iterations with a tolerance factor of $1 \mathrm{E}-3$.

Table. 1. Parameters used for flow simulation.

\begin{tabular}{|c|c|c|}
\hline Parameter & Symbol & Value assigned \\
\hline average pore diameter & $\mathrm{d}_{\mathrm{a}}$ & $10 \mu \mathrm{m}$ \\
\hline electrical conductivity & $\mathrm{k}$ & $3.4 \mathrm{e}-5 \mathrm{~S} / \mathrm{m}$ \\
\hline zeta potential & $\xi$ & $-0.1 \mathrm{~V}$ \\
\hline temperature & $T$ & $298 \mathrm{~K}$ \\
\hline
\end{tabular}

\section{Results and Discussion}

Fig. 2 shows the electroosmotic flow velocity contour plots with arrow plots showing the direction of flow for the four cases of porosity. The plot shows that an increase in the porosity near the wall region increases the flow rate in that region. However, the effect of this rise in the central region is found to be very small at the interface of the two regions which can be of significant amount if the domain is scaled down to micro level. This kind of increase will eventually enhance the device performance in which such variations of porosity are used.

Also, the results show that this will also compensate the effects of reduction in the porosity due to application of 
external pressure or any other effect near the boundary region. By careful examination of the visualized plots, it becomes clear that the there is an increase in electroosmotic velocity in the central region of constant porosity as the difference in porosity becomes higher in the two distinct regions.

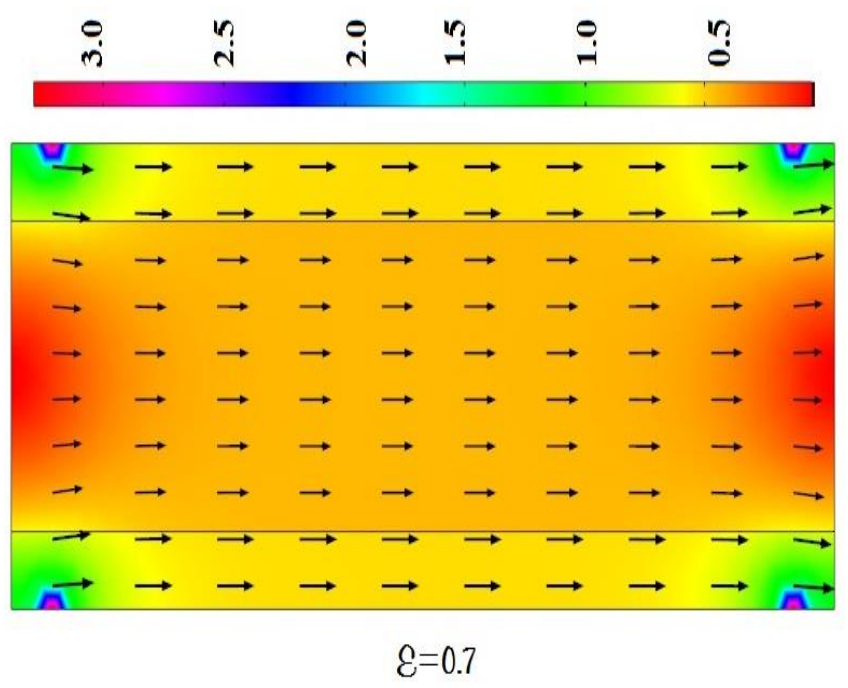

(a)

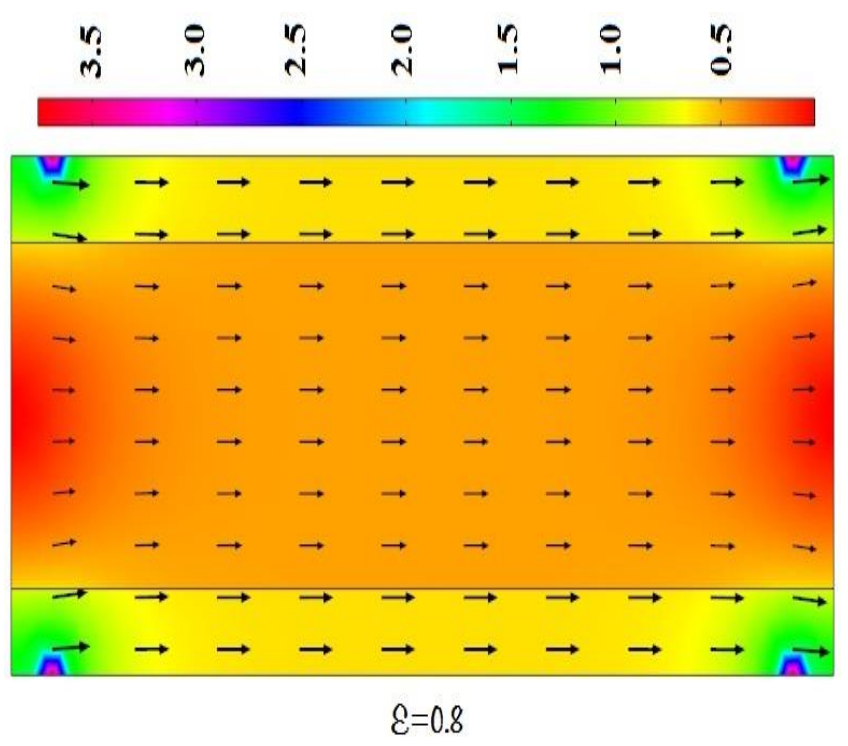

(b)

Fig. 2. Contour plots for electroosmotic flow velocity magnitude (units: $\mathrm{mm} / \mathrm{s}$ ); (a) Porosity $=0.7$, (b) Porosity $=0.8$.

\section{Conclusion}

In the present study, a numerical simulation has been carried out addressing the issue of reduction in porosity of porous layers adjacent to the solid wall present in an electroosmotic pump. This study emphasizes the need of a composite porous layer with a high porosity in the near wall region than the central region. The results show an appreciable increase in velocity magnitude especially near wall region but also in the central region. This increase in velocity has also been found out prominently for the case of pressure driven flows.

\section{Acknowledgment}

This work was supported by a National Research Foundation of Korea (NRF) grant funded by the Korea Government (MEST) (No. 2012R1A2A2A01046099), and a grant from the Priority Research Centers Program through the National Research Foundation of Korea (NRF) funded by MEST (2012-0005856).

\section{References}

(1) B. C. Chandrasekhara, D. Vortmeyer and Miinchen "Flow Model for Velocity Distribution in Fixed Porous Beds Under Isothermal Conditions" Thermo- and Fluid Dynamics, Vol. 12, pp. 105- 111, 1979.

(2) K. Vafai and C. L. Tien, "Boundary and inertia effects on flow and heat transfer in porous media" Int. J. of Heat Mass Transfer, Vol. 24, pp. 195-203, 1981.

(3) M. W. Kozak, E. J. Davis, "Electrokinetics of concentrated suspensions and porous media: I. Thin electrical double layers", Journal of Colloid and Interface Science, Vol. 127, pp. 497-510, 1989.

(4) M. W. Kozak, E. J. Davis, "Electrokinetics of concentrated suspensions and porous media: 2 . Moderately thick electrical double layers", Journal of Colloid and Interface Science, Vol. 129, pp. 166-174, 1989.

(5) A. Rathore, C. Horvath, "Capillary electrochromatography: theories on electroosmotic flow in porous media", Journal of Chromatography A, Vol. 781, pp. 185-195, 1997.

(6) M. Wang, S. Chen, Electroosmosis in homogeneously charged micro-and nano-scale random porous media, Journal of Colloid and Interface Science, Vol. 314, pp. 264-273, 2007. 
(7) S. Yao \& J. G. Santiago, "Porous glass electroosmotic pumps: theory" J. of colloid and Interface Science, Vol. 268, pp. 133-142, 2003. 